OPEN ACCESS

Edited by:

Bruno Christ,

Leipzig University, Germany

Reviewed by:

Roger David Soberanis Mukul, Technical University of Munich,

Germany

Vijay Rajagopal,

The University of Melbourne, Australia

${ }^{*}$ Correspondence:

Miroslav Jiř́k

miroslav.jirik@lfp.cuni.cz

Specialty section:

This article was submitted to

Computational Physiology and

Medicine,

a section of the journal

Frontiers in Physiology

Received: 20 June 2021

Accepted: 31 August 2021

Published: 01 October 2021

Citation:

Jiř́k M, Hácha F, Gruber I, Pálek R,

Mirka H, Zelezny M and Liška V (2021)

Why Use Position Features in Liver

Segmentation Performed by

Convolutional Neural Network.

Front. Physiol. 12:734217.

doi: 10.3389/fphys.2021.734217

\section{Why Use Position Features in Liver Segmentation Performed by Convolutional Neural Network}

\author{
Miroslav Jiřík ${ }^{1,2,3 *}$, Filip Hácha ${ }^{1,4}$, Ivan Gruber ${ }^{1,2}$, Richard Pálek ${ }^{3,5}$, Hynek Mírka ${ }^{3,6}$, \\ Milos Zelezny ${ }^{1}$ and Václav Liška ${ }^{3,5}$
}

${ }^{1}$ Department of Cybernetics, Faculty of Applied Sciences, University of West Bohemia, Pilsen, Czechia, ${ }^{2}$ New Technologies for the Information Society, Faculty of Applied Sciences, University of West Bohemia, Pilsen, Czechia, ${ }^{3}$ Biomedical Center, Faculty of Medicine in Pilsen, Charles University, Pilsen, Czechia, ${ }^{4}$ Department of Informatics, Faculty of Applied Sciences, University of West Bohemia, Pilsen, Czechia, ${ }^{5}$ Department of Surgery, University Hospital and Faculty of Medicine in Pilsen, Charles University, Pilsen, Czechia, ${ }^{6}$ Department of Radiology, University Hospital and Faculty of Medicine in Pilsen, Charles University, Pilsen, Czechia

Liver volumetry is an important tool in clinical practice. The calculation of liver volume is primarily based on Computed Tomography. Unfortunately, automatic segmentation algorithms based on handcrafted features tend to leak segmented objects into surrounding tissues like the heart or the spleen. Currently, convolutional neural networks are widely used in various applications of computer vision including image segmentation, while providing very promising results. In our work, we utilize robustly segmentable structures like the spine, body surface, and sagittal plane. They are used as key points for position estimation inside the body. The signed distance fields derived from these structures are calculated and used as an additional channel on the input of our convolutional neural network, to be more specific U-Net, which is widely used in medical image segmentation tasks. Our work shows that this additional position information improves the results of the segmentation. We test our approach in two experiments on two public datasets of Computed Tomography images. To evaluate the results, we use the Accuracy, the Hausdorff distance, and the Dice coefficient. Code is publicly available at: https://gitlab.com/hachaf/liver-segmentation.git.

Keywords: liver volumetry, semantic segmentation, machine learning, convolutional neural network, medical imaging, position features

\section{INTRODUCTION}

3D medical imaging methods are an essential tool in diagnostics and treatment strategy selection. Magnetic Resonance Imaging is available in all major hospitals, and the Computed Tomography (CT) is available even in smaller ones. The analysis of the image data is traditionally done by the expert-radiologist. During this procedure, the 3D data are usually viewed on a $2 \mathrm{D}$ monitor as slices. This makes the process complicated and time-consuming, even for a trained operator.

Semantic segmentation is a task where each pixel in an input image is classified into a specific class, i.e., per-pixel image classification. In the previous two decades, along with developments in the field of computer vision, semi-automatic and automatic liver segmentation tools based on traditional computer vision have been proposed (Moghbel et al., 2018) and many complex Computer-Aided diagnostic systems were introduced (Christ et al., 2017). However, despite all the 
effort, the problem is not been perfectly solved till today. The main problem is that in a CT image, the intensity of the liver is similar to the intensity of adjacent tissues. Therefore, solely based on the intensity, it is impossible to decide which tissue belongs to the liver and which belongs to another organ. Another challenge is the variability of the liver shape.

Since 2012, when AlexNet by Krizhevsky et al. (2012) dominated ImageNet challenge (Deng et al., 2009), the popularity of the traditional computer vision methods decreases at expenses of neural networks. Most of the recent approaches for semantic segmentation are based on Convolutional Neural Networks (CNNs) (for example, Long et al., 2015; Chen et al., 2017a,b), nevertheless, from 2020 Transformer (Vaswani et al., 2017; Dosovitskiy et al., 2020) based architecture become increasingly popular (for example, Carion et al., 2020; Zheng et al., 2021). Their main disadvantages are the necessity of big amount of training data, and their high computational complexity.

In medical imaging, U-Net (Ronneberger et al., 2015) is the golden standard architecture for semantic segmentation. A nested version of U-Net utilizes authors in Zhou et al. (2018). In the work (Radiuk, 2020), the author proposes 3D U-Net to perform multi-organ segmentation. In recent papers, the authors also experiment with transformers (Valanarasu et al., 2021), and their combination with the U-Net architecture (Chen et al., 2021). In our work, we decided to utilize the standard UNet architecture with some modifications tailored specifically to our task.

The standard procedure in machine learning is the normalization of the data. Removing the data variability by normalization makes learning more straightforward because the algorithm can be focussed on the simpler problem. In image processing, the most common normalization algorithms are oriented on the object's intensity, size, or position. In CT images, the position normalization is done by the standard pose of the patient, while the calibration of the device provides the intensity normalization.

In this paper, we take a step beyond the limit of the standard CT normalization. We focus on the use of robustly segmentable tissues in the human body, and we show that the precise knowledge of the position of these structures can be used for a relative position determination in the body. Furthermore, this information can be used with benefit for the automatic segmentation of the liver.

The typical size of an abdominal CT image is about $512 \times 512$ $\times 100$, which gives us 26.214 .400 voxels. It is hardly possible to train a neural network with such massive input. The usual way to solve this problem is to split the input image into smaller parts. The two most common attempts to solve this problem are separating the $2 \mathrm{D}$ slices or splitting the $3 \mathrm{D}$ input image into smaller blocks. However, by this procedure, some information is lost by the cropping operation. Each small slice or block of image data contains essential information about the surrounding tissues, but the position context is missing.

We suggest inserting additional position information into the training procedure. In our work, we used an adapted algorithm from the bodynavigation Python package (Jirik and Liska, 2018) to extract the robustly segmentable tissues and the Signed

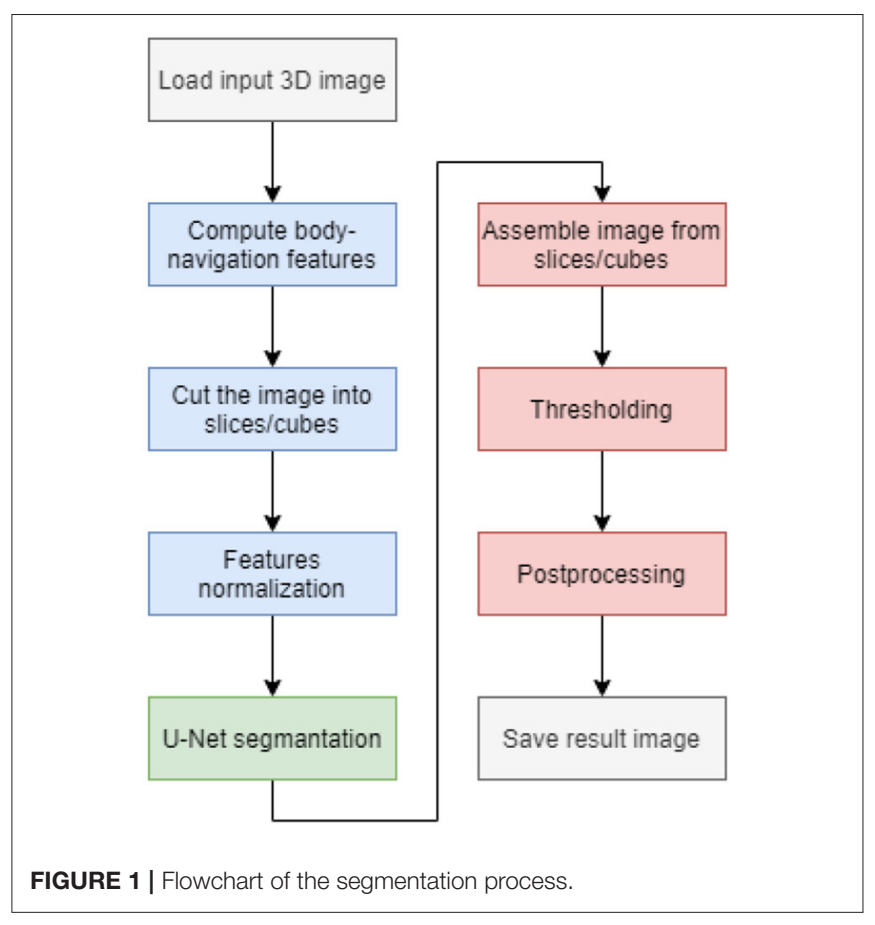

Distance Field (SDF) to each of these segmentations. During the training procedure, The SDFs are attached to the intensity image as additional channels. We tested this setup for two different segmentation approaches and show that it improves the segmentation results for both of them.

\section{PROPOSED METHOD}

Our segmentation method is based on a convolutional neural network with U-Net architecture. In this work, we tested two different approaches. The first approach is based on processing a single $2 \mathrm{D}$ slice from the original $3 \mathrm{D}$ volumetric image using standard U-Net architecture. This approach uses a discrete convolution of two-dimensional signals. Volumetric image is processed slice by slice, and the individual solutions are then assembled back into the original 3D image.

The second approach is based on the use of 3D signal convolution. The volumetric input image is cut into cubes of size $32 \times 32 \times 32$ voxels to overcome memory limits. These cubes are then processed by the neural network and then assembled back into the original 3D image once again. When preparing the training data, these cubes were cut using an offset of 8,16 , and 24 voxels in each direction, significantly increasing the amount of training data. For this approach, all 2D convolution layers in UNet are replaced by $3 \mathrm{D}$ convolution layers, as well as max-pooling layers. The rest of the architecture remains the same.

In addition to the neural network, the entire segmentation process is complemented by data normalization, feature space expansion using body-navigation features, and postprocessing of neural network output. The diagram of the whole process is shown in Figure 1. 


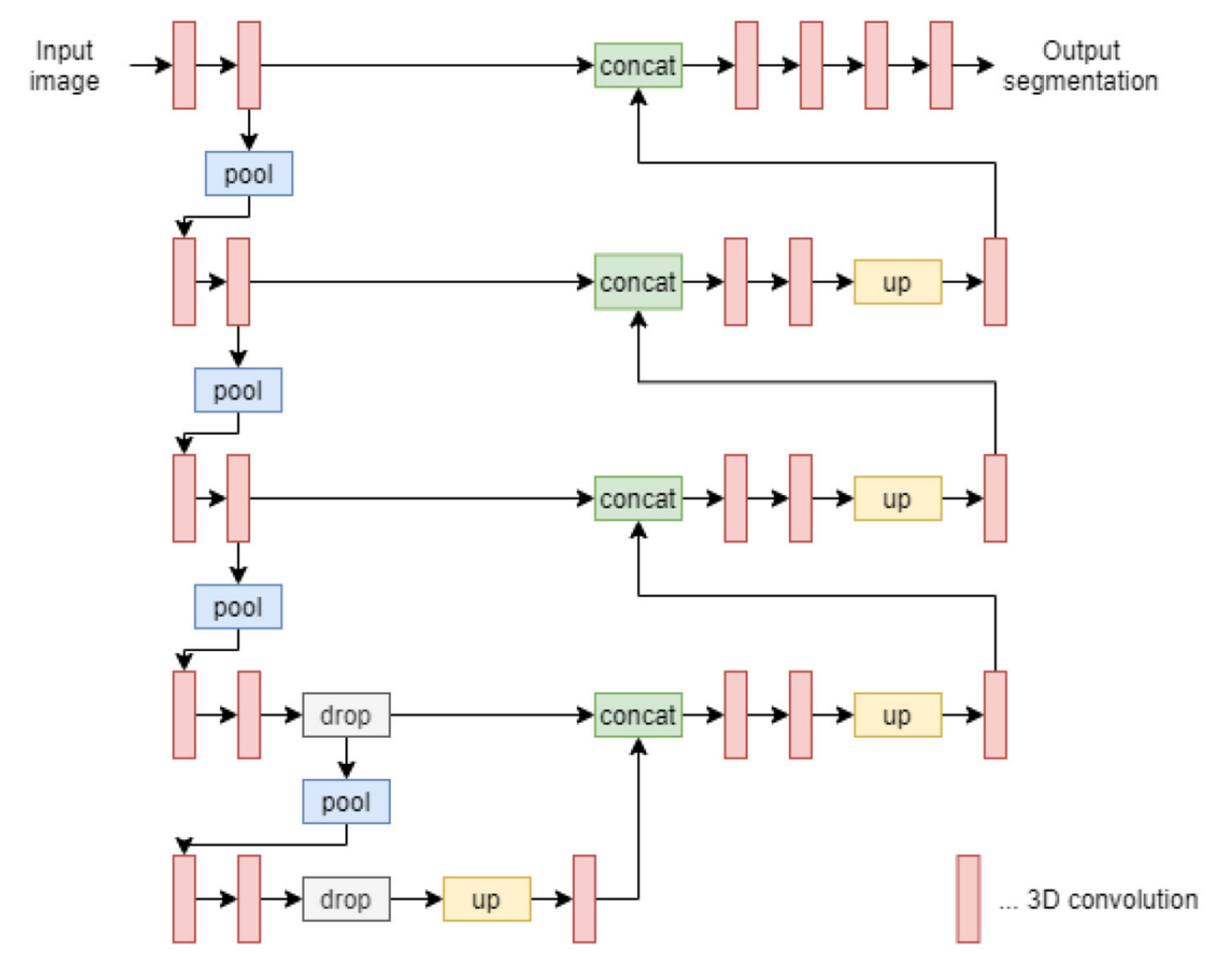

FIGURE 2 | U-Net 3D architecture diagram.

\subsection{Data Normalization}

Before the segmentation itself, we need to normalize the input CT image. The measured X-ray intensities given by the absorption of various tissue types are already partially normalized at the output of the CT scanner. Therefore, we only rescale them to the interval $[0,1]$ using standard min-max normalization (see Equation 1). The same normalization method is applied to the body-navigation features.

$$
x_{\text {norm }}=\frac{x-x_{\min }}{x_{\max }-x_{\min }}
$$

\subsection{U-Net}

The U-Net architecture consists of a contracting path and an expansive path. The contracting path is formed by repeating convolutional layers, followed by a rectified linear unit (ReLU) and a max-pooling layer for downsampling. The expansive path consists of an upsampling of the feature map followed by a convolution layer, a concatenation with the correspondingly cropped feature map from the contracting path, and two convolution layers, each followed by a ReLU. To avoid overfitting the network, we add dropout layers after the fourth and fifth levels of the contracting path.

To create a U-Net working with $3 \mathrm{D}$ cubes, it is necessary to replace $2 \mathrm{D}$ convolution layers with $3 \mathrm{D}$ convolution layers, as well as replace 2D max-pooling layers and 2D upsampling layers with their 3D counterparts. In other aspects, the architecture remains the same. A diagram describing the architecture is shown in Figure 2 .

\subsection{Body-Navigation Features}

The extraction of the body-navigation features is based on robustly segmentable structures which are accessible to segment, and its position in the body is stable - bones, body surface, and lungs (see Figure 3). We used previously introduced algorithms (Jirik and Liska, 2018) from the bodynavigation package for Python. In addition, we fine-tuned the algorithms for the sake of robustness. The first step is the image resize to a lower resolution for the sake of speed. Generally, for most features, the next step is segmentation, followed by SDF construction.

The body surface is extracted by thresholding of filtered image. A used threshold (parameter TB) is -300 [HU](Hounsfield Units), and a Gaussian filter does the filtration with a standard deviation (parameter $S B$ ) of $3 \mathrm{~mm}$.

The detection of the sagittal plane starts with the localization of the spine.

Gaussian filter does it with a standard deviation (of $100 \mathrm{~mm}$ in the axial (or transversal) plane and $15 \mathrm{~mm}$ in the perpendicular directions (parameter SS). It is applied on volumetric data thresholded by value 320[HU] (parameter TS). Then the center of the body surface and the center of the spine is used to construct a vector that defines the first estimation of the sagittal plane. In the following step, the $2 \mathrm{D}$ projection of bones into an axial plane is constructed. Then the mirror image of this projection is prepared. Finally, the symmetry is found by the iterative minimalization of the difference of $2 \mathrm{D}$ projection and its rotated and translated mirror image. 


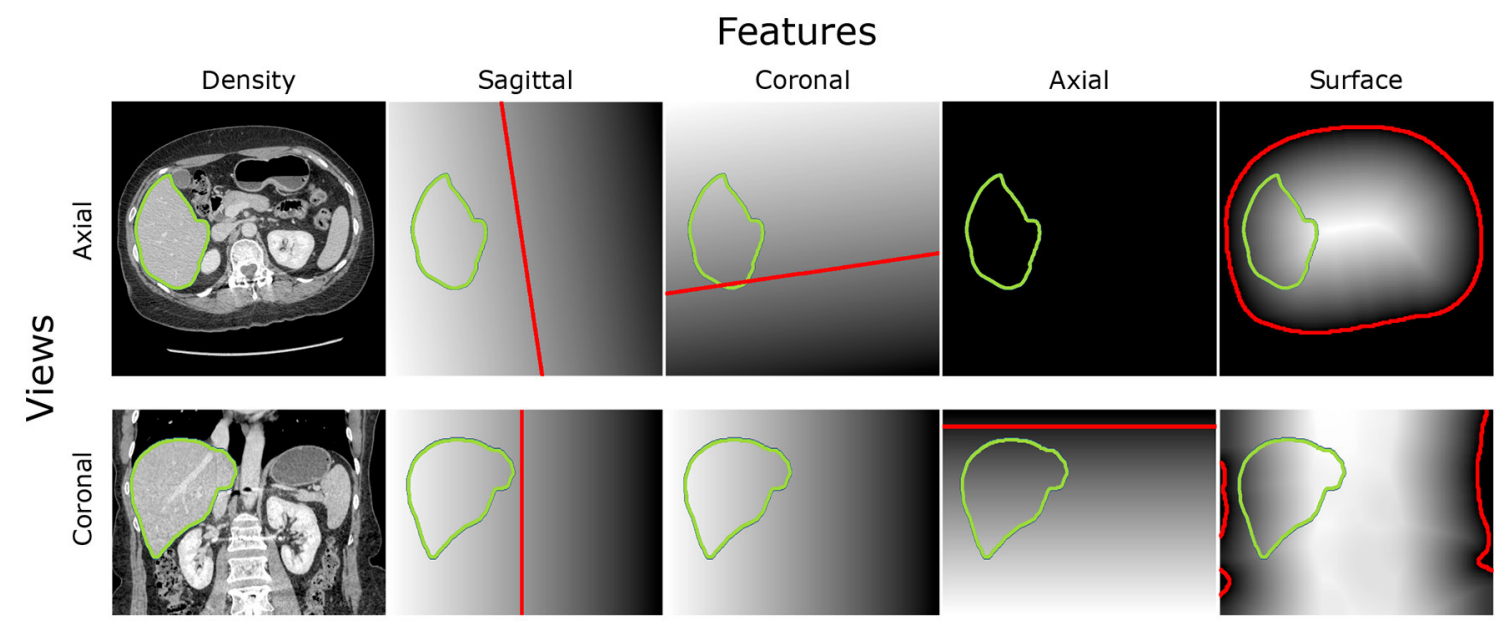

FIGURE 3 | Intensity features and position features based on robustly segmentable structures: The axial view on the middle slice of the CT scan can be found in the top row, while the coronal view is in the second row. The first column is the intensity image. The other columns show the Signed Distance Field (SDF) to the sagittal plane, coronal plane, the axial plane on the bottom level of the heart, and the body surface. The zero level of the SDF is red. The border of the liver is green.
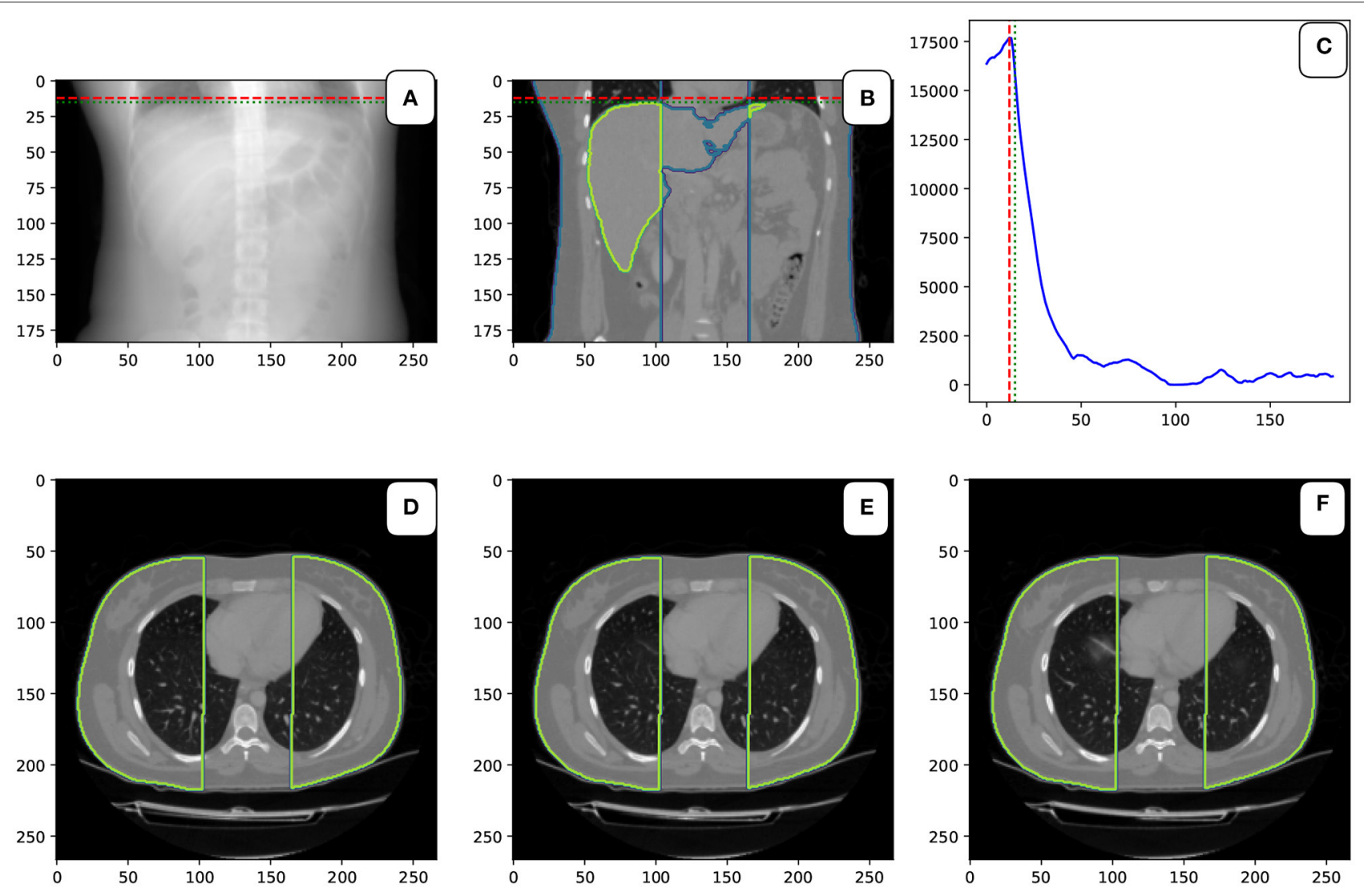

FIGURE 4 | Localization of the top of the diaphragm: Image (A,B) in the first row show the CT scan's coronal view. The green and blue delineation show the liver and the mask. The red dashed line is the detected level of the top of the diaphragm. The green dashed line shows the top level of the liver. The image (C) in the first row is the plot with the area of the masked cavity in square $\mathrm{mm}$. Images (D-F) show three consecutive axial slides of the CT scan. In the middle (E) is the slice from the top level of the diaphragm can. On the left (D) is the slice above the diaphragm. The right image (F) shows the slide under the top level of the diaphragm. A small "shadow" of the diaphragm can be seen in this image. 
The coronal plane is the plane perpendicular to the sagittal plane, and it is going through the spine. Thus, the orientation of SDF is given by the position of the center of the body surface.

We introduce the reference point in the craniocaudal axis, located at the top of the diaphragm. The localization of the point is based on the analysis of the series of slides. The internal cavity is defined by the density lower than -200 [HU] (parameter TLA). For each slide, the area of the internal cavity was measured. The areas closer than $30 \mathrm{~mm}$ to the sagittal plane (parameter DSA) and closer than $1 \mathrm{~mm}$ to the body surface (parameter $D B A$ ) are excluded from the calculation. This mask can be seen in the bottom row in Figure 4.

\subsection{Postprocessing}

After performing segmentation using a neural network, we obtain an output image formed by one channel with the same dimension as the input of the neural network. The values of the output image are in the range from 0 to 1 . This output is further processed. First, thresholding is performed with a threshold value $T h=0.5$.

The image is then divided into contiguous areas, and their volume is measured for each of them, given the number of voxels.
The area with the largest volume is left in the image, and the others are overwritten into the background.

We use mathematical morphology for further processing of the resulting image. The main reason for including this step was to simplify the boundary of the resulting object and to remove high-frequency noise. In the search for a suitable sequence of morphological operations on the test dataset, the best results were obtained by repeated use of four binary erosions.

\section{EXPERIMENTS}

To compare the benefits of usage of the body-navigation features in liver segmentation, we designed and implemented two experiments, one using U-Net 2D and one using U-Net 3D. In both experiments, the results of segmentation using the bodynavigation features were compared with the results achieved without their use.

Section 3.1 describes the data on which the neural networks were trained and used to validate and test the segmentation model. In section 3.2 we describe metrics used to evaluation of the segmentation results. In section 3.3, we describe the

TABLE 1 | Segmentation methods comparison on validation and test dataset.

\begin{tabular}{|c|c|c|c|c|c|c|c|}
\hline \multirow[b]{2}{*}{ Image } & \multirow[b]{2}{*}{ Body-nav. } & \multicolumn{3}{|c|}{ 2D model } & \multicolumn{3}{|c|}{ 3D model } \\
\hline & & Accuracy & MaxD & Dice & Accuracy & MaxD & Dice \\
\hline 3Dircadb-15 & No & 0.9898 & 50.8189 & 0.9094 & 0.1340 & 279.6009 & 0.1025 \\
\hline 3Dircadb-16 & No & 0.9773 & 52.0738 & 0.8713 & 0.1610 & 305.2199 & 0.1498 \\
\hline 3Dircadb-17 & No & 0.9773 & 54.6727 & 0.8755 & 0.1610 & 269.8953 & 0.1498 \\
\hline Sliver07-16 & No & 0.9831 & 37.2269 & 0.8912 & 0.1877 & 316.4408 & 0.1944 \\
\hline Sliver07-17 & No & 0.9911 & 224.5562 & 0.8361 & 0.1414 & 253.8510 & 0.1158 \\
\hline Sliver07-18 & No & 0.9849 & 250.1329 & 0.7539 & 0.1359 & 284.2670 & 0.1059 \\
\hline 3Dircadb-18 & No & 0.9732 & 79.9842 & 0.8892 & 0.1528 & 335.1785 & 0.1356 \\
\hline 3Dircadb-19 & No & 0.9678 & 71.5489 & 0.7918 & 0.1032 & 406.9116 & 0.0453 \\
\hline 3Dircadb-20 & No & 0.9851 & 50.0576 & 0.8815 & 0.1032 & 304.3232 & 0.0453 \\
\hline Sliver07-19 & No & 0.9616 & 118.8727 & 0.7853 & 0.1504 & 274.8742 & 0.1315 \\
\hline Sliver07-20 & No & 0.9731 & 245.2699 & 0.7165 & 0.1141 & 274.7924 & 0.0660 \\
\hline Avg. value & No & 0.9786 & 131.9546 & 0.8240 & 0.1404 & 300.4868 & 0.1129 \\
\hline St. dev. & No & 0.0092 & 85.0098 & 0.0646 & 0.0250 & 40.3931 & 0.0445 \\
\hline 3Dircadb-15 & Yes & 0.9871 & 34.0230 & 0.8897 & 0.9746 & 100.6067 & 0.7981 \\
\hline 3Dircadb-16 & Yes & 0.9706 & 31.3055 & 0.8118 & 0.9576 & 88.0981 & 0.7201 \\
\hline 3Dircadb-17 & Yes & 0.9780 & 53.7383 & 0.8799 & 0.9675 & 128.1953 & 0.8191 \\
\hline Sliver07-16 & Yes & 0.9818 & 33.5785 & 0.8801 & 0.9545 & 90.1366 & 0.7766 \\
\hline Sliver07-17 & Yes & 0.9931 & 45.1112 & 0.8695 & 0.9633 & 83.2325 & 0.7538 \\
\hline Sliver07-18 & Yes & 0.9928 & 151.0889 & 0.8645 & 0.9565 & 139.8558 & 0.6247 \\
\hline 3Dircadb-18 & Yes & 0.9761 & 41.7075 & 0.8964 & 0.9680 & 93.3783 & 0.7934 \\
\hline 3Dircadb-19 & Yes & 0.9721 & 46.9303 & 0.8146 & 0.9868 & 69.3535 & 0.7698 \\
\hline 3Dircadb-20 & Yes & 0.9817 & 48.7038 & 0.8584 & 0.9872 & 99.6428 & 0.7317 \\
\hline Sliver07-19 & Yes & 0.9693 & 57.9692 & 0.8209 & 0.9624 & 83.0969 & 0.7634 \\
\hline Sliver07-20 & Yes & 0.9829 & 187.7416 & 0.7968 & 0.9860 & 65.3701 & 0.8196 \\
\hline Avg. value & Yes & 0.9816 & 75.4617 & 0.8509 & 0.9695 & 94.6333 & 0.7609 \\
\hline St. dev. & Yes & 0.0083 & 52.1915 & 0.0353 & 0.0118 & 21.4375 & 0.0528 \\
\hline
\end{tabular}

All the values are without post-processing. 
training setup for the experiments, and finally, in section 3.4, we present the results obtained in our experiments using the above approaches. The source code used to perform these experiments is located on the LiverSegmentation repository.

\subsection{Dataset}

For our experiments, the dataset was composed of two public datasets. The Sliver07 dataset has been created by The Medical Image Computing and Computer-Assisted Intervention Society (MICCAI) for liver segmentation challenge (Heimann et al., 2009). In this dataset, the liver is outlined by a radiological expert in 20 CT scans. The other dataset is 3Dircadb by Soler (2016), and it was created by Research Institute against Digestive Cancer (IRCAD). It also contains $20 \mathrm{CT}$ scans and liver segmentations made by the radiologist. The number of slices in each series is from 64 to 515 . Slice thickness and pixel spacing vary from 0.5 to 5.0 and from 0.54 to $0.87 \mathrm{~mm}$, respectively. By the combination of these datasets, we get $5,7772 \mathrm{D}$ slices and $733,0373 \mathrm{D}$ cubes in total. For our experiments, the dataset was split into three parts-training, validation, and testing. The number of CT slices in training, validation, and testing is 24 , 6 , and 5, respectively. The entire dataset contains 564,200,960 voxels, while the liver, according to annotations, makes up only $7.72 \%$ of images. Segmented classes are therefore significantly unbalanced, so it is appropriate to use a weighted loss function (see section 3.3).

\subsection{Evaluation Metrics}

To compare the results of the individual segmentation methods, we used three commonly used metrics. The first metric is accuracy. This metric expresses the ratio of correctly classified voxels to the total number of voxels in the image. The formula for calculating this metric is shown in the Equation (2), where $T P$ is true positives, $T N$ is true negatives, $F P$ is false positives, and $F N$ is false negatives. This metric is often used not only in segmentation but also in classification tasks. However, due to the unbalanced ratio of the number of voxels that contain liver and the total number of voxels, it is not very suitable. To be more specific, the ratio between voxels of the liver and the other voxels is approximately 1:16.816. However, we decided to list accuracy because it is the standard metric.

$$
\mathrm{Acc}=\frac{T P+T N}{T P+T N+F P+F N}
$$

As another metric, we used the Dice similarity coefficient (also known as Intersection over Union), which is more suitable for our segmentation task. It was independently developed by Dice (1945) and (Sørenson, 1948). Dice similarity coefficient measures the similarity between two sets of data $X$ and $Y$. In our case, these sets are formed by predicted segmentation and true segmentation. The calculation of this metric is described by the Equation (3).

$$
\text { Dice }=2 \frac{|X \cap Y|}{|X|+|Y|}
$$

In medical imaging of the parenchymatous organs, the position of the wrongly classified voxel is critical. It is not a big problem if the voxel is adjacent to the segmented organ. However, it is more severe if the voxel far from the liver is classified as a liver. For this reason, we also measured the Hausdorf distance (see Aspert et al., 2002). This metric measures the maximum distance between the surfaces of the actual segmentation and the predicted segmentation. The maximum distance between surfaces $\mathcal{S}_{1}$ and $\mathcal{S}_{2}$ can be calculated according to the Equation (4) where $d\left(\mathcal{S}_{1}, \mathcal{S}_{2}\right)$ is the distance from surface $\mathcal{S}_{1}$ to surface $\mathcal{S}_{2}$ and can be calculated with the Equation (5).

$$
\begin{gathered}
\operatorname{MaxD}=\max \left(d\left(\mathcal{S}_{1}, \mathcal{S}_{2}\right), d\left(\mathcal{S}_{2}, \mathcal{S}_{1}\right)\right) \\
d\left(\mathcal{S}_{1}, \mathcal{S}_{2}\right)=\max _{p \in \mathcal{S}_{1}}\left(d\left(p, \mathcal{S}_{2}\right)\right)
\end{gathered}
$$

\subsection{Training Setup}

For both neural networks ( $2 \mathrm{D}$ and $3 \mathrm{D}$ U-Net), the Adam optimizer with a learning rate of 0.01 was used for the training. Weighted binary cross-entropy was used as a loss function, where

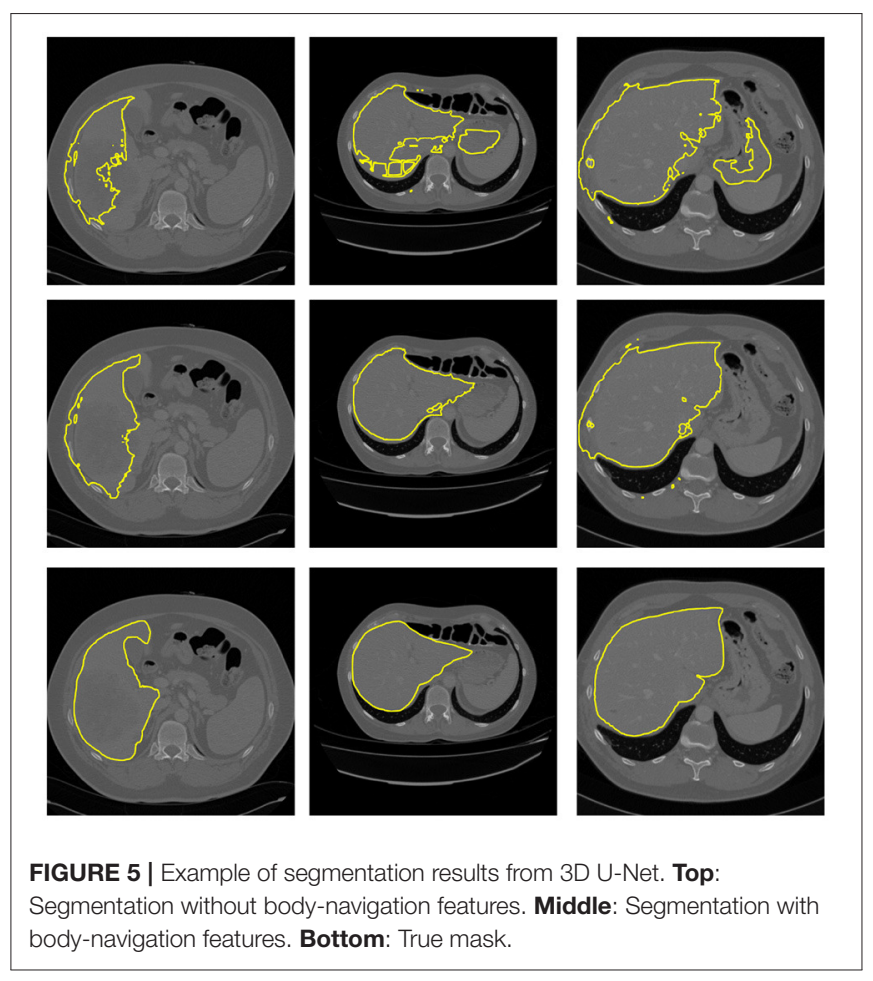

TABLE 2 | Evaluation of postprocessing in 3D segmentation model.

\begin{tabular}{llccc}
\hline & & \multicolumn{3}{c}{ 3D model } \\
\cline { 3 - 5 } Postprocessing & Body-nav. & Accuracy & MaxD & Dice \\
\hline No & No & 0.1404 & 300.4868 & 0.1129 \\
No & Yes & 0.9695 & 94.6333 & 0.7609 \\
Yes & No & 0.1552 & 292.2695 & 0.1237 \\
Yes & Yes & 0.9767 & 50.1791 & 0.7918
\end{tabular}


TABLE 3 | Sensitivity of the parameters for the positional features algorithm measured by relative displacement of the origin of the coordinate system: $k$ is the default parameter value multiplicator.

\begin{tabular}{|c|c|c|c|c|c|c|c|}
\hline \multirow[b]{2}{*}{$k$} & \multicolumn{7}{|c|}{ Error [mm] } \\
\hline & $25 \%$ & $50 \%$ & $75 \%$ & $100 \%$ & $125 \%$ & $150 \%$ & $175 \%$ \\
\hline Param (default value) & \multicolumn{7}{|c|}{ Reference } \\
\hline SB (3 [mm]) & 3.3002 & 2.7002 & 3.3002 & 0.0 & 10.5750 & 19.7250 & 24.9750 \\
\hline TB (-300 [HU]) & 80.2767 & 39.3823 & 10.9500 & 0.0 & 3.1501 & 3.9002 & 3.5253 \\
\hline TS (320 [HU]) & 77.9674 & 41.8941 & 31.0902 & 0.0 & 24.7194 & 42.3552 & 65.1209 \\
\hline SS $(100 \times 15 \times 15[\mathrm{~mm}])$ & 28.8292 & 22.3974 & 10.0019 & 0.0 & 21.7733 & 28.1594 & 31.8916 \\
\hline TLA (-200 [HU]) & 88.2000 & 25.4250 & 0.3750 & 0.0 & 0.6750 & 0.7500 & 1.2750 \\
\hline DBA (1 [mm]) & 0.0000 & 0.0000 & 0.0000 & 0.0 & 0.0000 & 0.2250 & 0.2250 \\
\hline DSA (30 [mm]) & 0.6750 & 0.6000 & 0.4500 & 0.0 & 2.7000 & 3.2250 & 5.1000 \\
\hline
\end{tabular}

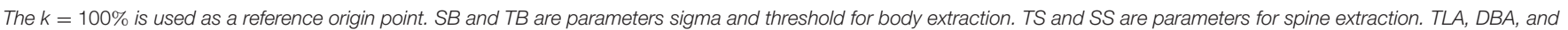
DSA are parameters for axial plane extraction.

the weights of the individual classes were adjusted inversely proportional to class frequencies in the input data. In the $2 \mathrm{D}$ experiments, the batch size was set to 32 and in the $3 \mathrm{D}$ experiments to 4 . In all experiments, the networks were trained for five epochs.

\subsection{Results}

The performed experiments can be divided into two groups$2 \mathrm{D}$ and $3 \mathrm{D}$ - according to the dimensionality of input data. In both of these groups, we performed the experiment with and without the body-navigation features. The measurements on the validation dataset and test dataset can be found in Table 1. An example of output segmentation is shown in Figure 5.

Based on measured data, it can be seen that the bodynavigation features enhance the quality of segmentation. The effect is stronger in the $3 \mathrm{D}$ experiment where the Dice coefficient increased noticeably. In both experimental setups, there has been a significant decrease in the Hausdorff distance. In the 3D setup, the improvement was much more significant once again.

The direct comparison of the 2D and the 3D neural network is inappropriate because, in the $2 \mathrm{D}$ setup, the whole slide gives a good idea about the relative position of each pixel in the human body. While using the 3D blocks, there is excellent information about the detail, but the spatial context is lost.

To evaluate the influence of the postprocessing step, the Table 2 contains all the metrics with and without postprocessing. The benefits of postprocessing are most evident in the measurement of the MaxD metric. The cause is the removal of incorrectly segmented small areas that are far from the segmented organ.

The position features can be generally used not only for liver segmentation. The CT density is not sufficient for the identification of the organ. I.g, the density of the liver is often similar to the density of the heart or spleen. The position of an organ is an important clue in the identification and segmentation process. We believe that there is potential benefit in using our positional feature-based algorithm to segment the other organs and anatomical structures in the abdominal cavity.
In our future work, it would be appropriate to consider the use of a combined segmentation method that would use a $3 \mathrm{D}$ convolution network working with local cubes as preprocessing for a 2D convolution network that would process entire 2D slices. Another possible approach would be to extend the input shape of the $3 \mathrm{D}$ convolutional network to include several whole $2 \mathrm{D}$ slices.

\subsection{Position-Features Sensitivity}

The position features algorithm is based on several parameters. To check the sensitivity to these parameters, we decided to include a test based on the stability of the point in the origin of the $3 \mathrm{D}$ space given by the sagittal plane, coronal plane, and the top level of the diaphragm. The default parameter value was multiplied by the parameter value multiplicator $k$. Then the position of the origin point obtained with default parameters is compared to the origin point obtained with the parameter multiplied with $k$. The origin of the coordinance system obtained by $k=100 \%$ is used as a reference.

Table 3 shows the mean error for all the investigated parameters over 20 images of the Ircadb1 dataset. It can be seen that the input parameters can be changed in a wide range with no significant impact on the result. The only exception is the threshold used for surface extraction. However, because of the intensity calibration of the CT data and the high contrast between the body and the air, it is easy to set it correctly. The example of the position features extracted with changed parameters can be found in Figure 6.

\section{CONCLUSION}

Our paper presents a method for incorporating position information into automatic liver segmentation performed by a neural network. In addition, we introduced a novel feature for the estimation of the position in the craniocaudal axis. To show the effect of the positional information, we perform experiments utilizing two different data structures. The experiments showed that the position information could significantly enhance the final segmentation quality. Furthermore, the effect is even more 


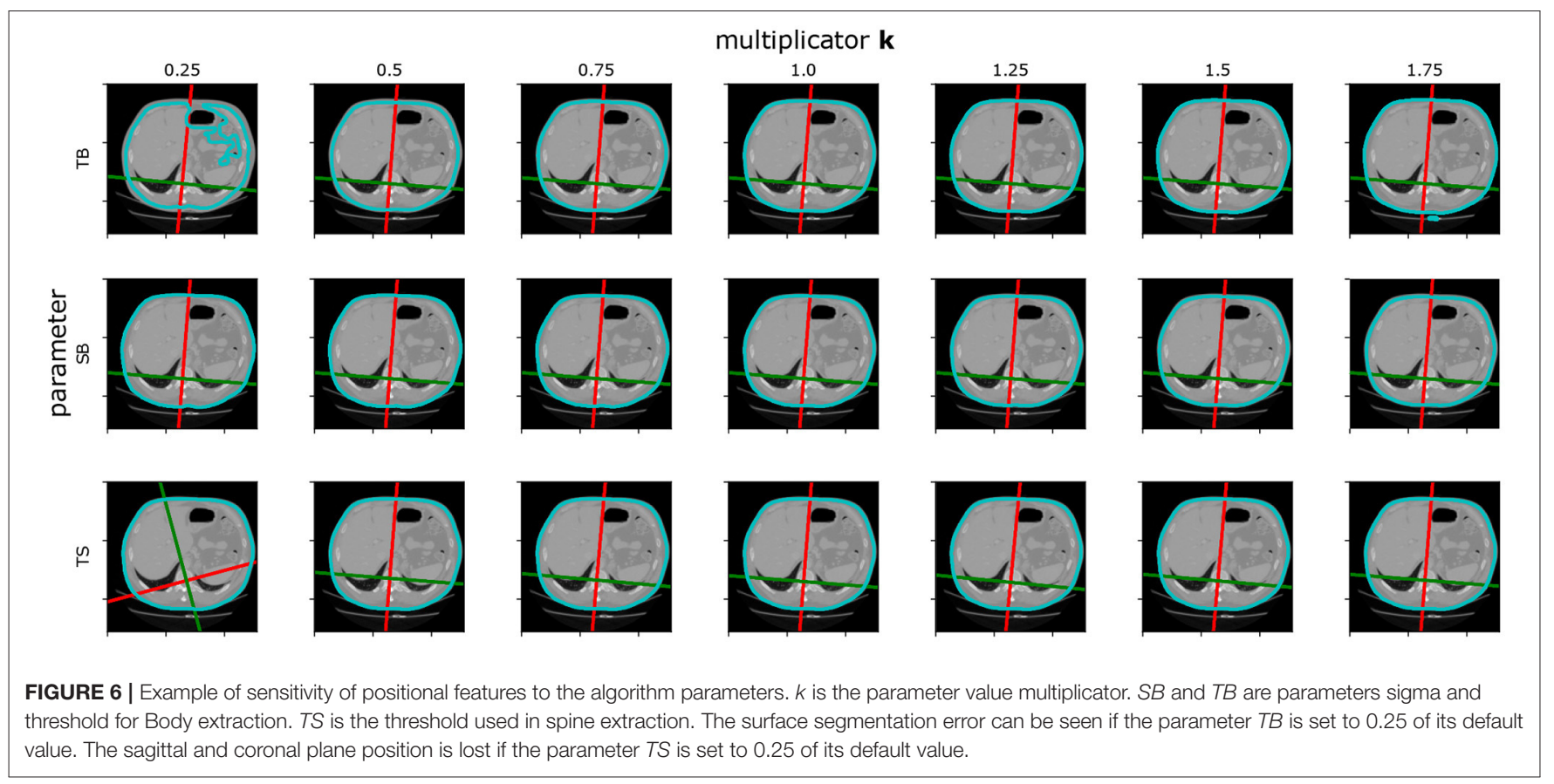

substantial if the training data are split into smaller blocks where the spatial context is hidden beyond their borders.

In our future work, we would like to prove the possibilities of using the positional features to segment other organs in the abdominal cavity. Furthermore, we plan to combine both tested approaches and test other possible data processing, especially in the $3 \mathrm{D}$ domain.

\section{DATA AVAILABILITY STATEMENT}

Publicly available datasets were analyzed in this study. This data can be found here: https://sliver07.grand-challenge.org/, https:// www.ircad.fr/research/3dircadb/.

\section{AUTHOR CONTRIBUTIONS}

MJ, FH, and VL conceived of the presented idea. MJ modified the positional features from the bodynavigation package, suggested a new diaphragm-based feature, and wrote important parts of the manuscript. FH designed and implemented the $3 \mathrm{D}$ processing pipeline and the related experiment, the author of the related manuscript parts, collected the data, performed the annotations, and wrote the section Introduction and data preparation paragraphs. IG provided the consultations on

\section{REFERENCES}

Aspert, N., Santa-Cruz, D., and Ebrahimi, T. (2002). "Mesh: measuring errors between surfaces using the Hausdorff distance," in Proceedings. IEEE International Conference on Multimedia and Expo (Lausanne). doi: 10.1109/ICME.2002.1035879
Convolutional Neural Networks, contributed to the sections Introduction and the Conclusion of the manuscript, and made the final proofreading. MZ, VL, RP, and HM provided critical reading and suggested important changes in the manuscript, also provided critical feedback, and helped shape the research, analysis, and manuscript. All authors contributed to the article and approved the submitted version.

\section{ACKNOWLEDGMENTS}

This work was supported by the Ministry of Education of the Czech Republic, project No. LTARF18017, and by Charles University Research Center program UNCE/MED/006 University Center of Clinical and Experimental Liver Surgery and Ministry of Education project ITI CZ.02.1.01/0.0/0.0/17_048/ 0007280: Application of modern technologies in medicine and industry. The research was also supported by the project LO 1506 of the Czech Ministry of Education, Youth and Sports. The work was supported by the grant SVV-2020-2022 No 260 535. The authors appreciate the access to computing and storage facilities owned by parties and projects contributing to the National Grid Infrastructure MetaCentrum provided under the program Projects of Large Research, Development, and Innovations Infrastructures (CESNET LM2015042). S. (2020). End-to-End object detection with transformers. arXiv preprint arXiv:2005.12872. doi: 10.1007/978-3-030-58452-8_13

Chen, J., Lu, Y., Yu, Q., Luo, X., Adeli, E., Wang, Y., et al. (2021). Transunet: Transformers make strong encoders for medical image segmentation. arXiv preprint arXiv:2102.04306. 
Chen, L.-C., Papandreou, G., Kokkinos, I., Murphy, K., and Yuille, A. L. (2017a). Deeplab: Semantic image segmentation with deep convolutional nets, atrous convolution, and fully connected CRFs. IEEE Trans. Pattern Anal. Mach. Intell. 40, 834-848. doi: 10.1109/TPAMI.2017.2699184

Chen, L.-C., Papandreou, G., Schroff, F., and Adam, H. (2017b). Rethinking atrous convolution for semantic image segmentation. arXiv preprint arXiv: 1706.05587

Christ, B., Dahmen, U., Herrmann, K. H., König, M., Reichenbach, J. R., Ricken, T., et al. (2017). Computational modeling in liver surgery. Front. Physiol. 8:906. doi: $10.3389 /$ fphys.2017.00906

Deng, J., Dong, W., Socher, R., Li, L.-J., Li, K., and Fei-Fei, L. (2009). "ImageNet: a large-scale hierarchical image database," in 2009 IEEE Conference on Computer Vision and Pattern Recognition (Miami, FL: IEEE), 248-255. doi: 10.1109/CVPR.2009.5206848

Dice, L. R. (1945). Measures of the amount of ecologic association between species. Ecology 26, 297-302. doi: 10.2307/1932409

Dosovitskiy, A., Beyer, L., Kolesnikov, A., Weissenborn, D., Zhai, X., Unterthiner, T., et al. (2020). An image is worth 16x16 words: transformersfor image recognition at scale. arXiv preprint arXiv:2010.11929.

Heimann, T., van Ginneken, B., Styner, M. A., Arzhaeva, Y., Aurich, V., Bauer, C., et al. (2009). Comparison and evaluation of methods for liver segmentation from CT datasets. IEEE Trans. Med. Imaging 28, 1251-1265. doi: 10.1109/TMI.2009.2013851

Jirik, M., and Liska, V. (2018). "Body navigation via robust segmentation of basic structures," in VipIMAGE 2017: Proceedings of the VI ECCOMAS Thematic Conference on Computational Vision and Medical Image Processing Porto (Portugal), 306-314. doi: 10.1007/978-3-319-68195-5_33

Krizhevsky, A., Sutskever, I., and Hinton, G. E. (2012). Imagenet classification with deep convolutional neural networks. Adv. Neural Inform. Process. Syst. 25, 1097-1105. doi: 10.1145/3065386

Long, J., Shelhamer, E., and Darrell, T. (2015). "Fully convolutional networks for semantic segmentation," in Proceedings of the IEEE Conference on Computer Vision and Pattern Recognition, 3431-3440. doi: 10.1109/CVPR.2015.7298965

Moghbel, M., Mashohor, S., Mahmud, R., and Saripan, M. I. B. (2018). Review of liver segmentation and computer assisted detection/diagnosis methods in computed tomography. Artif. Intell. Rev. 50, 497-537. doi: 10.1007/s10462-017-9550-x

Radiuk, P. (2020). Applying 3D U-Net architecture to the task of multi-organ segmentation in computed tomography. Appl. Comput. Syst. 25, 43-50. doi: 10.2478/acss-2020-0005

Ronneberger, O., Fischer, P., and Brox, T. (2015). "U-Net: convolutional networks for biomedical image segmentation," in International Conference on Medical
Image Computing and Computer-Assisted Intervention (Munich: Springer), 234-241. doi: 10.1007/978-3-319-24574-4_28

Soler, L. (2016). 3D-IRCADb-01. Available online at: http://www.ircad.fr/research/3d-ircadb-01/

Sørenson, T. (1948). A Method of Establishing Groups of Equal Amplitude in Plant Sociology Based on Similarity of Species Content and Its Application to Analyses of the Vegetation on Danish Commons. Biologiske skrifter. I kommission hos E. Munksgaard.

Valanarasu, J. M. J., Oza, P., Hacihaliloglu, I., and Patel, V. M. (2021). Medical transformer: gated axial-attention for medical image segmentation. arXiv preprint arXiv:2102.10662.

Vaswani, A., Shazeer, N., Parmar, N., Uszkoreit, J., Jones, L., Gomez, A. N., et al. (2017). "Attention is all you need," in Advances in Neural Information Processing Systems, eds. S. J. Hanson, J. D. Cowan, and C. L. Giles (San Francisco, CA: Morgan Kaufmann Publishers Inc.) 5998-6008.

Zheng, S., Lu, J., Zhao, H., Zhu, X., Luo, Z., Wang, Y., et al. (2021). "Rethinking semantic segmentation from a sequence-to-sequence perspective with transformers," in Proceedings of the IEEE/CVF Conference on Computer Vision and Pattern Recognition, 6881-6890.

Zhou, Z., Siddiquee, M. M. R., Tajbakhsh, N., and Liang, J. (2018). "UNet++: a nested U-Net architecture for medical image segmentation," in Deep Learning in Medical Image Analysis and Multimodal Learning for Clinical Decision Support (Springer), 3-11. doi: 10.1007/978-3-030-00889-5_1

Conflict of Interest: The authors declare that the research was conducted in the absence of any commercial or financial relationships that could be construed as a potential conflict of interest.

Publisher's Note: All claims expressed in this article are solely those of the authors and do not necessarily represent those of their affiliated organizations, or those of the publisher, the editors and the reviewers. Any product that may be evaluated in this article, or claim that may be made by its manufacturer, is not guaranteed or endorsed by the publisher.

Copyright (C) 2021 Jiř́k, Hácha, Gruber, Pálek, Mírka, Zelezny and Liška. This is an open-access article distributed under the terms of the Creative Commons Attribution License (CC BY). The use, distribution or reproduction in other forums is permitted, provided the original author(s) and the copyright owner(s) are credited and that the original publication in this journal is cited, in accordance with accepted academic practice. No use, distribution or reproduction is permitted which does not comply with these terms. 